\title{
Detection of Trichomonas vaginalis in Vaginal Swab Clinical Samples from Palestinian Women by Culture
}

\author{
Yasmeen Houso, ${ }^{1}$ Mohammad A. Farraj, ${ }^{1}$ As'ad Ramlawi, ${ }^{2}$ and Tamer Essawi ${ }^{1}$ \\ ${ }^{1}$ Master Program in Clinical Laboratory Science, Birzeit University, P.O. Box 14, Birzeit, Palestine \\ ${ }^{2}$ Central Public Health Laboratory, Palestinian Ministry of Health, Ramallah, West Bank, Palestine \\ Correspondence should be addressed to Mohammad A. Farraj, mfarraj@birzeit.edu
}

Received 26 September 2011; Accepted 1 November 2011

Academic Editors: M. Canica and M. C. Lai

Copyright (C) 2011 Yasmeen Houso et al. This is an open access article distributed under the Creative Commons Attribution License, which permits unrestricted use, distribution, and reproduction in any medium, provided the original work is properly cited.

\begin{abstract}
Background. Trichomoniasis is a common sexually transmitted disease caused by Trichomonas vaginalis. It is a major health problem worldwide. The World Health Organization (WHO) has estimated that 180 million infections are acquired annually worldwide. Methodology. Vaginal swabs (1207) were cultured for T. vaginalis on Trichomonas Medium no. 2 (Oxoid) soon after specimen collection. The cultures were examined daily using a light microscope to detect the presence of T. vaginalis. Results. The prevalence of T. vaginalis was $13.6 \%$ (164/1207). The infection rate was the highest during pregnancy, $28.1 \%$, and the lowest among women whose spouses use condoms, $8.6 \%$. Conclusions. The culture method was used in this study to accurately determine the prevalence of this parasite in the West Bank, Palestine. The results of the study will eliminate ambiguities concerning trichomoniasis in this country and will contribute to better management and proper treatment.
\end{abstract}

\section{Introduction}

Trichomoniasis, caused by Trichomonas vaginalis, is the most common sexually transmitted disease worldwide. According to the World Health Organization (WHO), an estimated 170 million new infections occur annually throughout the world [1]. Data on the prevalence and incidence rates of this parasite remains limited in many developing countries including Palestine [2]. According to syndromic diagnosis, the number of patients with trichomoniasis in Palestine in 2003 was 103 females and 36 males per 100,000 [3].

T. vaginalis is associated with diverse pregnancy outcomes and may serve as a cofactor in human immunodeficiency virus (HIV) transmission [4]. T. vaginalis increases the risk of HIV transmission as seen among African-Americans in the United States $[4,5]$. T. vaginalis causes a common genitourinary infection, which is frequently asymptomatic; about $10-50 \%$ of infections in women are asymptomatic [6]. In women, trichomoniasis is characterized by a yellow-green frothy vaginal discharge, vaginal odor, pain with sexual intercourse, pain during urination, and vulvovaginal soreness and itching [7]. Complications in pregnant women include postabortion infection, postcesarean infection, preterm birth, low birth weight infants, and preterm labor causing premature rupture of the membranes [7]. T. vaginalis infection has been suggested as a risk factor for developing cervical neoplasia [8]. Other complications caused by $T$. vaginalis include pelvic inflammatory disease and tubal infertility, vaginitis, and urethritis [9]. In males, it is usually found in the urethra, prostate or epididymis causing urethritis and prostatitis [10].

Traditionally, physicians diagnose trichomoniasis on the basis of the clinical picture. Symptoms alone are not sufficient to make reliable diagnosis of $T$. vaginalis infection. Laboratory tests such as wet mount are routinely performed for this purpose. Although rapid and inexpensive, this technique has a low sensitivity of 50 to $80 \%$ [11-13]. For accurate diagnosis by wet mount, adequate numbers of the parasite must be present and the characteristic tumbling motility must be observed by an experienced technician within a short time [10].

The culture method was used in this study because it is the "gold standard" against which the performance of other diagnostic methods is compared $[11,12]$. The aim of this 
study was to determine the prevalence of T. vaginalis among Palestinian women in the West Bank, Palestine. This is the first study conducted in this country that utilized the culture method and did not rely on direct microscopic examination.

\section{Materials and Methods}

2.1. Study Design and Target Population. This prospective study was conducted on women attending reproductive health clinics in the West Bank, Palestine. Unduplicated vaginal swabs were randomly collected by the attending physician from women residing in districts throughout the West Bank, Palestine. Informed consent was obtained from all women participating in the study.

2.2. Sample Collection and Processing. Samples were collected from 1207 women of childbearing age from October 2004 to December 2005. Vaginal discharge was taken from the posterior cervix or from the vaginal wall using a sterile swab containing Stuart's transport media (Via Magent 77/6, Rho (MI) Italy). The swabs were immediately transported to the Central Public Health Laboratory in Ramallah, Palestine and cultured within 4 hours after collection. Litmus paper was used to determine the $\mathrm{pH}$ of the discharge; the color change was compared with a $\mathrm{pH}$ standard color chart [14]. In most cases, a high vaginal $\mathrm{pH}(>4.5)$ is indicative of trichomoniasis.

The swabs were removed from the transport media and used to inoculate Trichomonas Medium no. 2 (Oxoid). The cultures were then incubated at $37^{\circ} \mathrm{C}$ and checked daily for motility under the microscope for a total of 5 days.

2.3. Statistical Analysis. Chi-square was used to analyze the data obtained in this study at 0.05 level of significance using SPSS 13 statistical package (SPSS Inc., Chicago, Ill).

\section{Results}

This study was conducted on a total of 1207 women of childbearing age attending reproductive health clinics in the West Bank, Palestine. The culture results revealed a prevalence of $13.6 \%$ (164/1207). The rate of positive cultures for $T$. vaginalis was $22.7 \%(5 / 22)$ in women of 18 years and younger and $6.8 \%(4 / 59)$ in women of 46 years and older. Several variables were taken into consideration during this study. These variables included age, marital status, pregnancy and lactation, use of contraceptives, socioeconomic conditions, education, and symptoms. These results are summarized in Table 1.

The symptoms experienced by all women participating in this study are documented and summarized in Table 2. The percentage of positive cultures for $T$. vaginalis ranged from $13.1 \%$ (130/990) for vaginal discharge to $15.9 \%$ (48/302) for pelvic pain as shown in Table 2 .

\section{Discussion}

T. vaginalis is the third most common cause of vaginitis. According to the WHO, T. vaginalis accounts for approx- imately half of all curable sexually transmitted diseases worldwide [1]. Annually it affects 170 million women worldwide [1]. The objective of this study was to determine the prevalence of trichomoniasis among Palestinian women by using the culture method.

Vaginal trichomoniasis is a recognized sexually transmitted disease. Sexually active women are at an increased risk of vaginitis due to this parasite. In this study, the prevalence of T. vaginalis was $13.6 \%$. The prevalence of this parasite in Israel is $8.1 \%$ in patients with vaginitis [15]. Prevalence rates vary with different patient population and diagnostic methods. In this study, the percentage of positive cultures may correlate with sexual activity. As shown in Table 1, the percentage of positive cultures of $T$. vaginalis tends to decrease with advanced age as compared to young sexually active women. Although the number of women above 46 years of age was low (4/59), the low percentage of positive cultures of $6.8 \%$ may correlate with reduced sexual activity.

Interestingly, a study was conducted in Gaza (Palestine) using the Papanicolaou smears (Pap) to determine the prevalence of $T$. vaginalis among pregnant women [2]. The prevalence was $18.2 \%$ as compared to $28.1 \%$ obtained in this study. The Pap smears are not reliable and inaccurate for the diagnosis of $T$. vaginalis and have a low sensitivity of $61 \%$ [16] as compared to culture, the golden standard with a sensitivity of $80-95 \%$ and specificity exceeding $95 \%$.

Considering women status, trichomoniasis is unusual in lactating women, since the glycogen-poor atrophic vagina of the breastfeeding woman is relatively hostile to this parasite [17]. Comparing the percentage of positive cultures found for married women (13.6\%), pregnant women $(28.1 \%)$, and lactating women $(11.4 \%), \chi^{2}$ results were significant $(P<$ 0.05).

The use of contraceptives is effective in reducing the percentage of positive cultures. In this study, the results obtained did not reveal a statistical significance $(P>0.05)$ in the percentage of positive cultures by using different means of contraceptives. The percentage was low (8.6\%) in women whose spouses used condoms as compared to the others. This indicates that using barrier contraceptives (condoms) is the most effective mechanical barrier to various microorganisms [18]. Women using either barrier or oral contraceptives six months before becoming pregnant had decreased rates to be colonized by T. vaginalis as previously described [19].

Diagnosis of trichomoniasis is usually based on the presence of symptoms such as vaginal discharge, irritation, genital soreness, pelvic pain, burning sensation, and vaginal $\mathrm{pH}$. Although $93 \%$ of the women had a vaginal $\mathrm{pH}>4.5$ (1125/1207) and 82\% have vaginal discharge (990/1207), the percentage of positive cultures was $13.7 \%(154 / 1125)$ and 13.1\% (130/990), respectively, as shown in Table 2. T. vaginalis grows best at acidic environment and attaches itself by its axostyle thus creating the irritation, inflammation, and other symptoms associated with the infection by this parasite [17]. There were no significant differences in the percentage of positive cultures of $T$. vaginalis according to occupation, education, or economic status.

In conclusion, this study showed that the prevalence of T. vaginalis in the West Bank is $13.6 \%$. This relatively high 
TABLE 1: The frequency of positive cultures of T. vaginalis in 1207 women according to different variables.

\begin{tabular}{|c|c|c|c|c|}
\hline \multirow{2}{*}{ Variable } & \multirow[b]{2}{*}{ Number of cases } & \multicolumn{2}{|c|}{ Positive cultures } & \multirow{2}{*}{$\begin{array}{c}\chi^{2} \\
P \text { value }\end{array}$} \\
\hline & & Number & Percent & \\
\hline \multicolumn{5}{|l|}{ Age groups (years) } \\
\hline $15-18$ & 22 & 5 & 22.7 & 0.393 \\
\hline $19-25$ & 261 & 41 & 15.7 & \\
\hline $26-30$ & 255 & 35 & 13.7 & \\
\hline $31-35$ & 259 & 31 & 12 & \\
\hline $36-45$ & 307 & 43 & 14 & \\
\hline Over 46 & 59 & 4 & 6.8 & \\
\hline Totals & 1163 & 159 & 13.7 & \\
\hline \multicolumn{5}{|l|}{ Marital status } \\
\hline Married & 1197 & 163 & 13.6 & $>0.05$ \\
\hline Divorced & 3 & 0 & 0 & \\
\hline Widow & 7 & 1 & 14.3 & \\
\hline Totals & 1207 & 164 & & \\
\hline \multicolumn{5}{|l|}{ Women status } \\
\hline Pregnant & 178 & 50 & 28.9 & $<0.05$ \\
\hline Lactating & 324 & 37 & 11.4 & \\
\hline Other & 705 & 77 & 10.9 & \\
\hline Total & 1207 & 164 & 13.6 & \\
\hline \multicolumn{5}{|l|}{ Women contraceptive use } \\
\hline IUD & 458 & 53 & 11.6 & 0.791 \\
\hline Pills & 130 & 12 & 9.2 & \\
\hline Others (coitus interrupts) & 25 & 3 & 12.0 & \\
\hline Non-users & 594 & 96 & 16.2 & \\
\hline Totals & 1207 & 164 & 13.6 & \\
\hline \multicolumn{5}{|l|}{ Condom use } \\
\hline Condoms used & 81 & 7 & 8.6 & $<0.05$ \\
\hline Condoms not used & 1126 & 157 & 13.9 & \\
\hline Totals & 1207 & 164 & 13.6 & \\
\hline \multicolumn{5}{|l|}{ Occupation } \\
\hline Working women & 59 & 6 & 10.2 & 0.463 \\
\hline Housewives & 1130 & 155 & 13.7 & \\
\hline Totals & 1189 & 161 & 13.5 & \\
\hline \multicolumn{5}{|l|}{ Education } \\
\hline High school & 1084 & 150 & 13.8 & 0.451 \\
\hline College & 123 & 14 & 11.4 & \\
\hline Totals & 1207 & 164 & 13.6 & \\
\hline \multicolumn{5}{|l|}{ Socioeconomic class } \\
\hline Low & 542 & 78 & 14.4 & 0.462 \\
\hline Moderate & 646 & 83 & 12.8 & \\
\hline High & 19 & 3 & 15.8 & \\
\hline Totals & 1207 & 164 & 13.6 & \\
\hline
\end{tabular}

Notes: married women have a stable sexual partner. All pregnant women are married. Socioeconomic classes are determined based on the poverty line put by the Palestine Central Bureau of Statistics, family income, the level of education, and profession. 
TABle 2: Positive cultures of T. vaginalis according to symptoms. Total number of samples is 1207 .

\begin{tabular}{lcccc}
\hline \multirow{2}{*}{ Symptoms } & \multicolumn{2}{c}{ Rate of symptoms } & \multicolumn{2}{c}{ Positive for T. vaginalis } \\
& Number & $\%$ & Number & $\%$ \\
\hline $\mathrm{pH}>4$ & 1125 & 93.2 & 154 & 13.7 \\
Vaginal discharge & 990 & 82 & 130 & 13.1 \\
Urinary & 489 & 40.5 & 67 & 13.7 \\
symptoms & & & & \\
Irritation & 458 & 38 & 68 & 14.9 \\
Soreness & 382 & 31.7 & 57 & 14.9 \\
Pelvic pain & 302 & 25 & 48 & 15.9 \\
\hline
\end{tabular}

number of positive cultures is of public health concern. This study warrants having better understanding and more accurate evaluation of urogenital trichomoniasis in Palestine. To curb the high rates of trichomoniasis and help in its prevention and control among Palestinian people, it is recommended that the Ministry of Health must take drastic measures to implement effective screening programs, provide treatment for infected partners, and spread awareness in the population about this disease and its transmission. In addition, it is extremely important to screen and treat asymptomatic women who may have persistent trichomoniasis for years.

\section{References}

[1] World Health Organization, Global Prevalence and Incidence of Selected Curable Sexually Transmitted Infections: Overview and Estimates, World Health Organization, Geneva, Switzerland, 2001.

[2] A. I. Al-Hindi and A. H. Lubbad, "Trichonamas vaginalis infection among Palestinian women: prevalence and trends during 2000-2006," Turkish Journal of Medical Sciences, vol. 36, no. 6, pp. 371-375, 2006.

[3] Palestinian Ministry of Health, "Annual report," 2002, the status of health in Palestine.

[4] P. C. Guenthner, W. E. Secor, and C. S. Dezzutti, “Trichomonas vaginalis-induced epithelial monolayer disruption and human immunodeficiency virus type 1 (HIV-1) replication: implications for the sexual transmission of HIV-1," Infection and Immunity, vol. 73, no. 7, pp. 4155-4160, 2005.

[5] F. Sorvillo, L. Smith, P. Kerndt, and L. Ash, "Trichomonas vaginalis, HIV, and African-Americans," Emerging Infectious Diseases, vol. 7, no. 6, pp. 927-932, 2001.

[6] S. Mullick, D. Watson-Jones, M. Beksinska, and D. Mabey, "Sexually transmitted infections in pregnancy: prevalence, impact on pregnancy outcomes, and approach to treatment in developing countries," Sexually Transmitted Infections, vol. 81, no. 4, pp. 294-302, 2005.

[7] J. R. Schwebke and D. Burgess, "Trichomoniasis," Clinical Microbiology Reviews, vol. 17, no. 4, pp. 794-803, 2004.

[8] S. Khurana, N. M. Dubey, and N. Malla, "Association of parasitic infections and cancers," Indian Journal of Medical Microbiology, vol. 23, no. 2, pp. 74-79, 2005.

[9] T. L. Cherpes, H. C. Wiesenfeld, M. A. Melan et al., "The associations between pelvic inflammatory disease, Trichomonas vaginalis infection, and positive herpes simplex virus type 2 serology," Sexually Transmitted Diseases, vol. 33, no. 12, pp. 747-752, 2006.

[10] H. Swygard, A. C. Sena, M. M. Hobbs, and M. S. Cohen, "Trichomoniasis: clinical manifestations, diagnosis and management," Sexually Transmitted Infections, vol. 80, no. 2, pp. 91-95, 2004.

[11] T. Crucitti, E. van Dyck, A. Tehe et al., "Comparison of culture and different PCR assays for detection of Trichomonas vaginalis in self collected vaginal swab specimens," Sexually Transmitted Infections, vol. 79, no. 5, pp. 393-398, 2003.

[12] K. A. Borchardt and R. F. Smith, "An evaluation of an InPouch(TM) TV culture method for diagnosing Trichomonas vaginalis infection," Genitourinary Medicine, vol. 67, no. 2, pp. 149-152, 1991.

[13] L. Pattullo, S. Griffeth, L. L. Ding et al., "Stepwise diagnosis of Trichomonas vaginalis infection in adolescent women," Journal of Clinical Microbiology, vol. 47, no. 1, pp. 59-63, 2009.

[14] D. G. Ferris, S. L. Francis, E. D. Dickman, K. Miler-Miles, J. L. Waller, and N. McClendon, "Variability of vaginal $\mathrm{pH}$ determination by patients and clinicians," Journal of the American Board of Family Medicine, vol. 19, no. 4, pp. 368-373, 2006.

[15] M. Dan, N. Kaneti, D. Levin, F. Poch, and Z. Samra, "Vaginitis in a gynecologic practice in Israel: causes and risk factors," Israel Medical Association Journal, vol. 5, no. 9, pp. 629-632, 2003.

[16] T. T. Lobo, N. Feijo, S. E. Carvalho et al., "A comparative evaluation of the Papanicolaou test for the diagnosis of trichomoniasis," Sexually Transmitted Diseases, vol. 30, no. 9, pp. 694-699, 2003.

[17] D. Petrin, K. Delgaty, R. Bhatt, and G. Garber, "Clinical and microbiological aspects of Trichomonas vaginalis," Clinical Microbiology Reviews, vol. 11, no. 2, pp. 300-317, 1998.

[18] M. L. Shew, J. D. Fortenberry, W. Tu et al., "Association of condom use, sexual behaviors, and sexually transmitted infections with the duration of genital human Papillomavirus infection among adolescent women," Archives of Pediatrics and Adolescent Medicine, vol. 160, no. 2, pp. 151-156, 2006.

[19] N. K. Mahdi, Z. H. Gany, and M. Sharief, "Risk factors for vaginal trichomoniasis among women in Basra, Iraq," Eastern Mediterranean Health Journal, vol. 7, no. 6, pp. 918-924, 2001. 

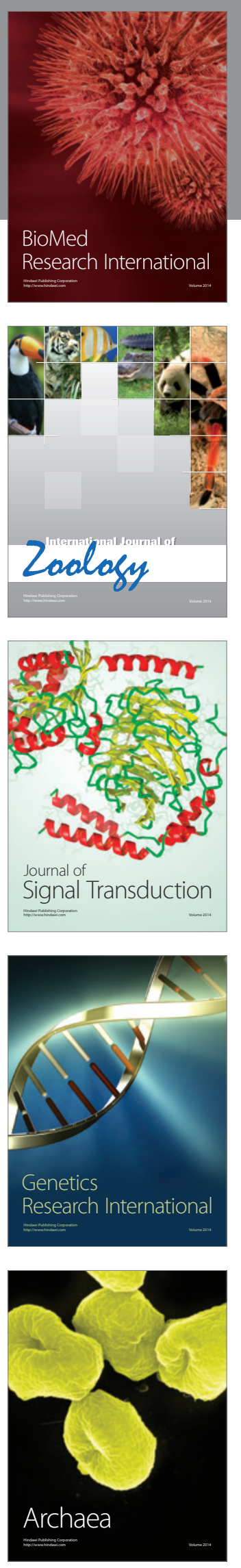
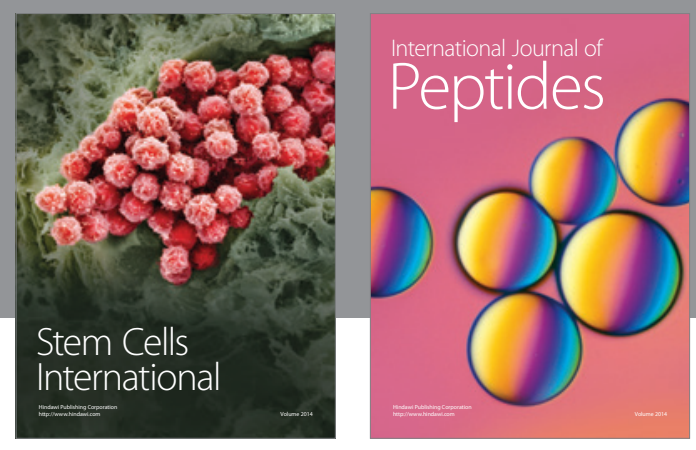

Submit your manuscripts at

http://www.hindawi.com
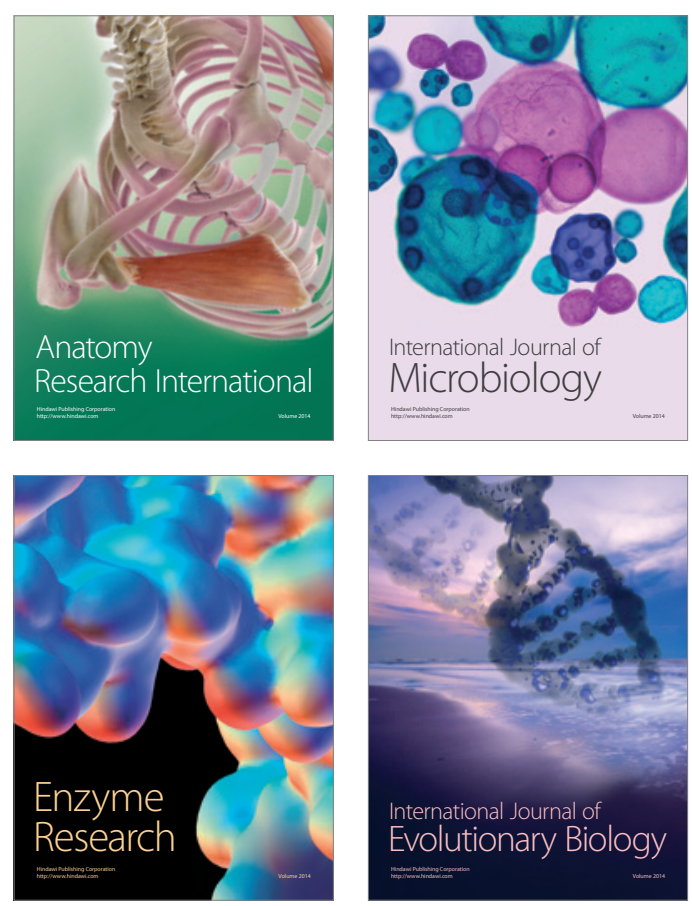
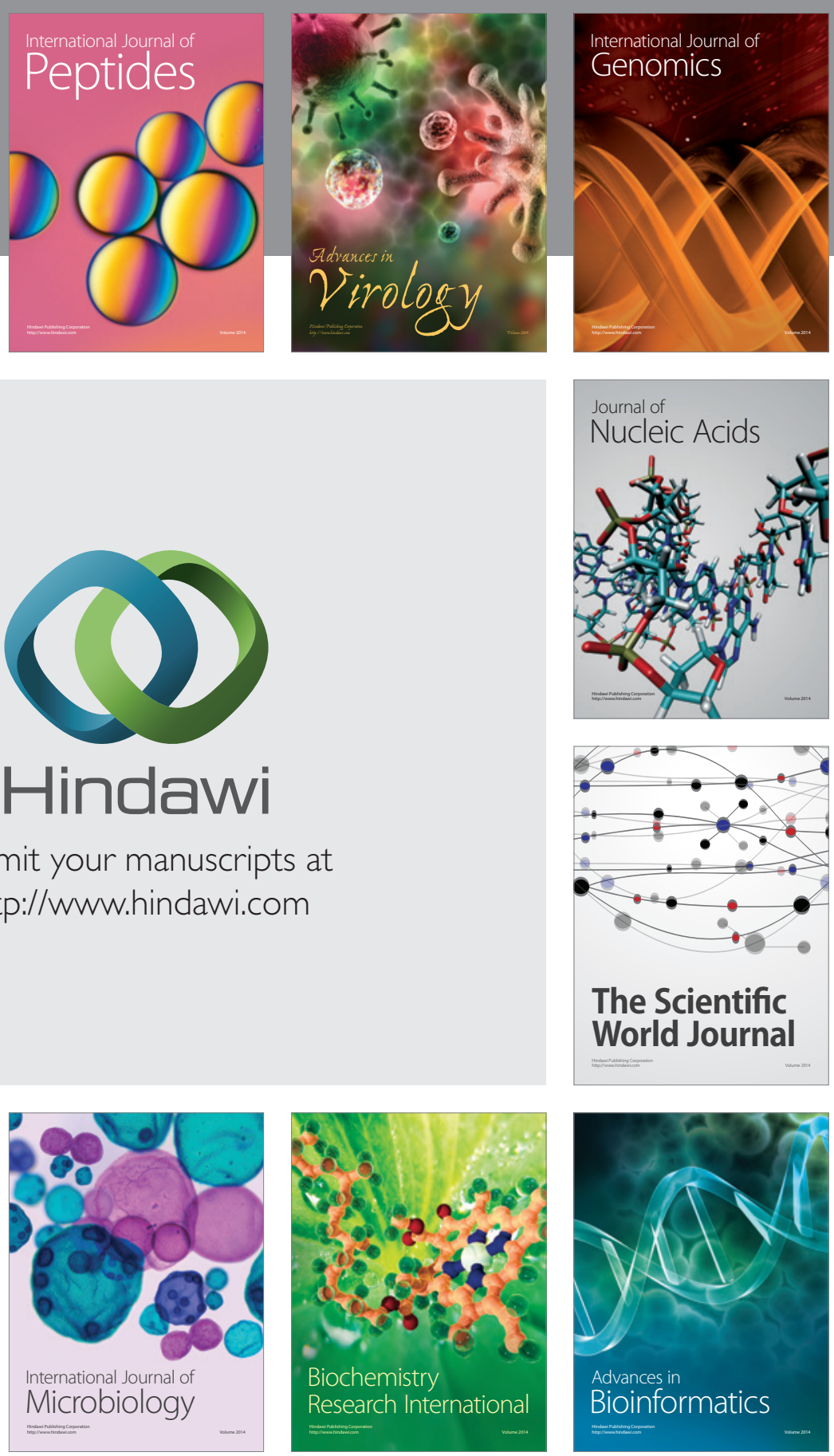

The Scientific World Journal
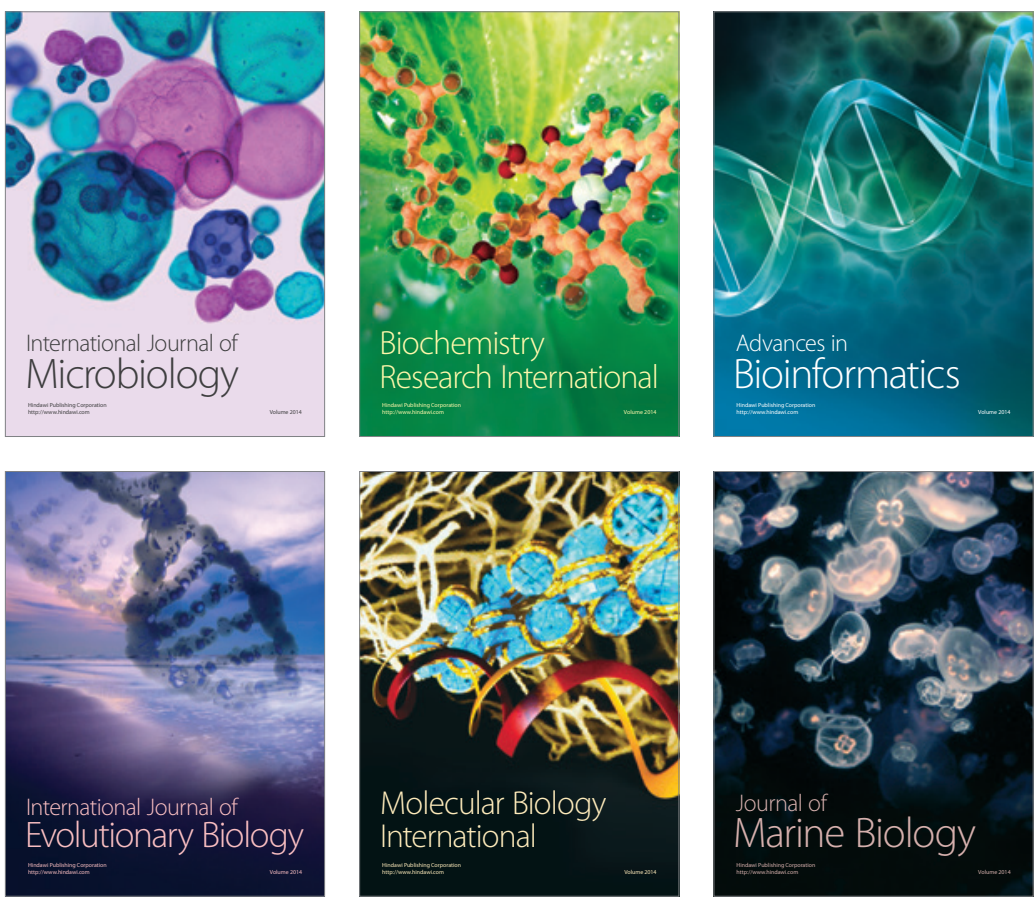